\title{
Cyclic Motion Control for Programmable Bevel-tip Needle 3D Steering: a Simulation Study
}

\author{
Eloise Matheson, Thomas Watts, Riccardo Secoli, and Ferdinando Rodriguez y Baena ${ }^{1}$
}

\begin{abstract}
Flexible, steerable, soft needles are desirable in Minimally Invasive Surgery to achieve complex trajectories while maintaining the benefits of percutaneous intervention compared to open surgery. One such needle is the multi-segment Programmable Bevel-tip Needle (PBN), which is inspired by the mechanical design of the ovipositor of certain wasps. PBNs can steer in 3D whilst minimizing the force applied to the surrounding substrate, due to the cyclic motion of the segments. Taking inspiration also from the control strategy of the wasp to perform insertions and lay their eggs, this paper presents the design of a cyclic controller that can steer a PBN to produce a desired trajectory in 3D. The performance of the controller is demonstrated in simulation in comparison to that of a direct controller without cyclic motion. It is shown that, while the same steering curvatures can be attained by both controllers, the time taken to achieve the configuration is longer for the cyclic controller, leading to issues of potential under-steering and longer insertion times.
\end{abstract}

\section{INTRODUCTION}

In Minimally Invasive Surgery (MIS) the ability to achieve complex tool trajectories, combined with the clinical advantages of percutaneous intervention [21] has led to the research field of flexible needles [6]. Needle insertions are highly prevalent in both diagnostic and intervention medical procedures, including biopsy, ablation, brachytherapy and fluid delivery and extraction. The success of these procedures is dependent on the precision and accuracy of the tip placement at the target position and orientation. These procedures can be aided by steerable devices, as such devices can accurately achieve target placement, increase the workspace of the needle, as well as avoid critical areas or obstacles along the way, including nerves, vessels and bones [15]. This is of particular importance in neurosurgery, where straight line trajectories can be undesirable for, e.g., deep brain stimulation due to anatomical reasons, as targets can be blocked by eloquent brain tissue [6].

Exhaustive reviews of steerable, flexible needles can be found in [16], [18]. Bevel-tip needles [13], pre-curved needles and concentric tube needles [20], [5] can achieve nonstraight paths by mechanical design, as curvature is achieved by the application of off-axis reaction forces at the needle tip from the resulting motion against tissue. 3D steering is achieved via rotation along the needle axis, however such movement can induce a torsional moment on the tissue [12], which can induce unwanted tissue damage. 3D steering can also be achieved by actuated needle designs, such as tendondriven needles [9] and shape-memory material designs [8].

\footnotetext{
${ }^{1}$ All authors are with the Mechatronics in Medicine Laboratory, Imperial College London, South Kensington, London, United Kingdom f.rodriguez@imperial.ac.uk
}

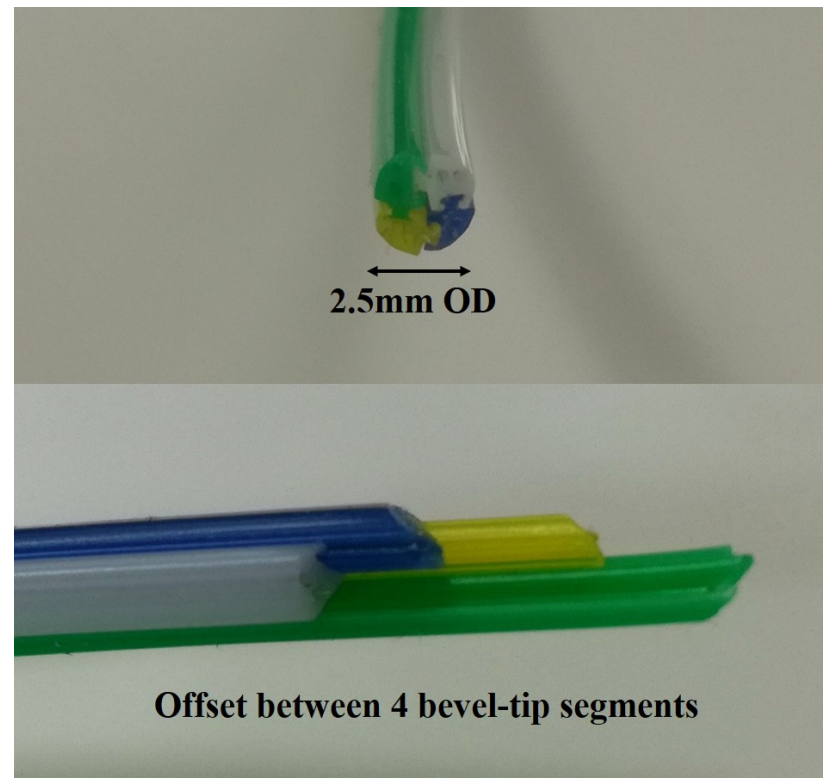

Fig. 1. Biocompatible clinically-sized Programmable Bevel-tip Needle

Whilst promising due to their steering performance, these systems have problems of miniaturization, sterilizability and mechanical complexity. To find a needle design that can steer in 3D without axial rotation, and without requiring complex actuation mechanisms, we can look to nature for inspiration, where evolution has already optimized the design of natural systems to perform complicated tasks. One such example are ovipositing wasps, whether parasitic (egg-laying in host larvae) or non-parasitic (eggs are inserted into some substrate, such as wood). These wasps can successfully penetrate and steer through a medium in order to lay eggs in a safe chamber. Their ovipositors - thin, flexible needlelike structures - can also avoid buckling whilst steering in 3D; two attractive traits for medical needles. The ovipositor consists of several longitudinally inter-connected chambers which can slide axially against one another, and steering is achieved by varying the distal offset of the valves via protracting one of the them at a time [4]. The chance of buckling is minimized through the use of directional serrations near the top of the ovipositor, which act as anchors against the substrate as a neighboring valve is advanced, creating stabilization and decreasing the axial push required for insertion. The cyclic motion, whereby one valve is pulled backwards and anchored, whilst the other is pushed further into the substrate, enables the forward motion to be achieved with a near-zero net force, placing no theoretical limit on its 


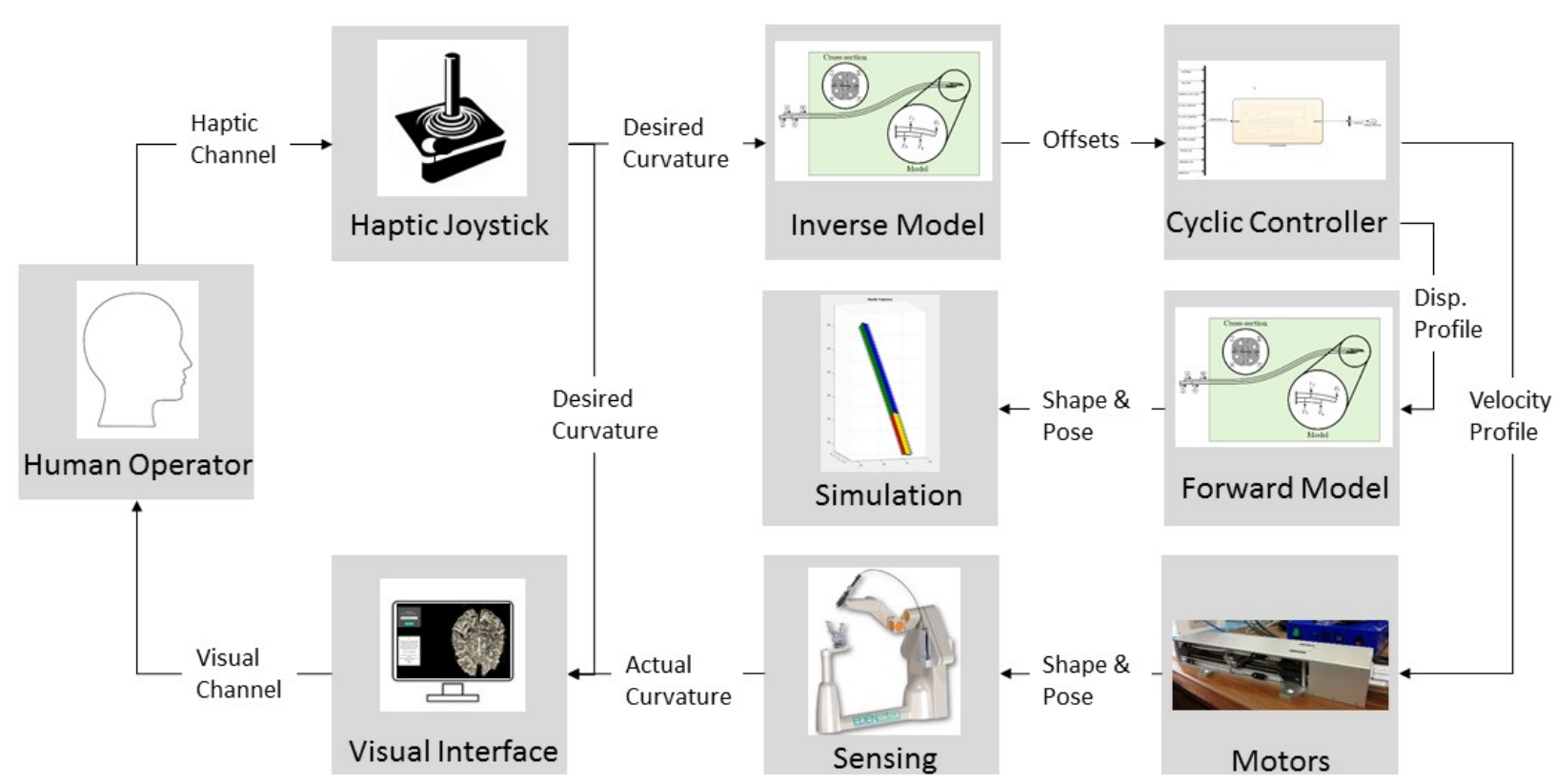

Fig. 2. System Architecture for Neurosurgical Procedures.

length [7]. The three segment fruit-fly parasitoid ovipositor was found to steer in any direction relative to their body orientation, always using a reciprocal motion profile when steering through a stiff substrate to minimize the net pushing force required [4].

The wasp ovipositor was the basis of inspiration for Programmable Bevel-tip Needles (PBNs). PBNs consist of multiple segments with bevel tips held together via an interlocking mechanism. The first PBN, codenamed STING, was presented in [7] and consisted of a 2 segment, $4.4 \mathrm{~mm}$ outer diameter (OD) rubber prototype, capable only of planar steering. Following design iterations and plastic rapidprototyping, 4 segment PBNs capable of 3D steering with $12 \mathrm{~mm}$ OD [10], $8 \mathrm{~mm}$ OD [2], and $4 \mathrm{~mm}$ OD [3] were presented. Finally a clinically acceptable, extruded biocompatible prototype of $2.5 \mathrm{~mm}$ OD was presented in [14]. Shown in Fig. 1, the PBN has an interlocking dove-tail mechanism. A PBN with a 7 segment, nitinol wire design [16] has also been developed where the rods are linked together via a tube holder.

Cyclic motion of the segments can minimize the required pushing force, which also decreases the displacement and strain applied on the surrounding tissue. This was shown to be true in simulation and in trials with laser reflective seeded phantom gels for planar steering [11], [14]. It is hypothesized that minimizing tissue strain minimizes tissue damage, which is the subject of ongoing clinical trials with sheep beginning this year. This paper extends the 2D cyclic controller design [14], and presents the first work towards the design of a 3D cyclic controller. Needle steering in 3D is demonstrated in simulation, and the performance of the controller is compared to that of a $3 \mathrm{D}$ direct controller.

\section{METHODOLOGY}

\section{A. Design}

The cyclic controller is designed with inspiration from the ovipositor cyclic motion profiles, where segments slide relative to one another in a back and forward patten, in order to minimize the pushing force required to part a substrate. For steering in $3 \mathrm{D}$, the controller needs to account for the motion of four different segments that make up the PBN. This low level cyclic controller fits into a larger architecture of needle insertion system, shown in Fig. 2.

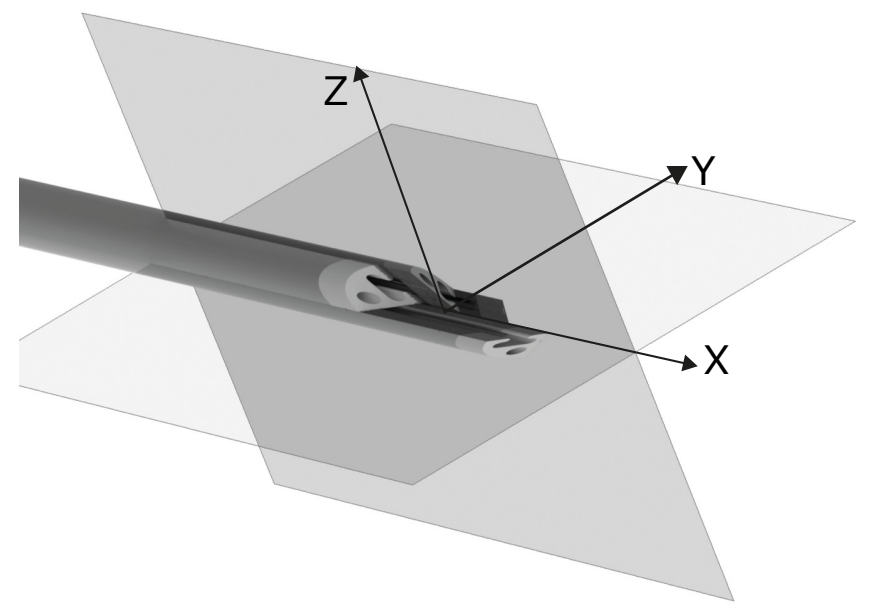

Fig. 3. A 4 segment probe with the curvature planes and axes

For a neurosurgical procedure, the system architecture is envisioned to include a human surgeon/operator, a joystick, models of the needle, a low-level controller, the mechatronic system and a visual interface. A desired path that the needle 
should follow will be pre-planned based on Magnetic Resonance Imaging (MRI) images and displayed to the operator via the visual interface. The path the operator then commands with the joystick is defined by two varying curvature values, defined in the Parallel Transport Frame (PTF) or Bishop's frame [1] of the needle (see Fig. 3). These curvature values are passed to the inverse model of the needle, which returns the required offsets between the needle segments to achieve that curvature. These required offsets serve as the inputs to the low-level controller, which generates the motion profiles (containing cyclic motions) for the motors to move the segments and create the commanded offset.

The expected curvature of the needle, based on the forward model of the needle converting segment offset to curvature, as well as the actual curvature of the needle (as measured by sensors including Fibre Bragg Gratings embedded within each segments and intra-operative 3D Ultrasound to detect the gross geometry) can then be shown to the operator via the visual interface, who can use the joystick to adjust the curvature commands, closing the control loop. The display of actual and commanded curvature is necessary as the nonholonomic constraints and kinematics of the needle mean it is not possible for the needle to instantaneously change curvature. By visually showing the two different paths (actual and commanded), the operator will also be informed to leave sufficient time for the system to update before overcorrecting the commanded curvature. This paper specifically integrates the Inverse Model, Cyclic Controller, Forward Model and Simulation modules shown in system architecture in Fig. 2.

A 3D linear inverse model, presented in [17] and based on an unmanned, aerial vehicle, is used to approximate the relationship between desired curvature and segment offsets via an optimization function. These segment offsets are then sent to both a low level cyclic controller which determines the velocity and displacement profiles for the motor to follow, as well as a low level direct controller which determines the velocity and displacement profiles when no cyclic motion is applied. To compare the performance of the low level controllers, the segment displacement configuration is sent to a forward model that converts segment offsets to achieved curvature and simulates the path taken. This forward model is based on multi-segment beam deformation and was first presented in [19].

The cyclic controller is largely based on the work presented in [14] with the following key improvements:

1) Offset commands between all four segments can now be processed (rather than just one offset which was previously used for planar steering) to achieve 3D steering.

2) The profile window is reduced to 2 seconds (rather than 8 seconds) which increases the cyclic controller frequency.

3) The algorithm is interruptible when the target point is reached by any segment within a predefined resolution.

\section{B. Simulation}

The controllers have been implemented in SIMULINK and MATLAB 2017b ${ }^{\circledR}$ (MathWorks Inc). New cyclic and direct profiles, based on the current desired offset from the inverse model module, are generated every 2 seconds. Our previous work [14] used an 8 second window however recommended this window be shortened to have a faster control frequency and input processing rate. The trade off is that the cyclic profile occurs in less time, resulting in shorter movements which can lead to less smooth motion. For this reason, shorter windows than 2 seconds were discarded, and 2 seconds chosen in simulation to analyze the response. The needle has a net velocity of $1 \mathrm{~mm} / \mathrm{s}$, such that there would be a constant insertion into the brain during a neurosurgical procedure. This design has been chosen from feedback from a neurosurgical advisory board who expressed a preference that a predictable and automatic insertion speed during a real procedure would be desirable, as opposed to the option of manual speed control. Offsets between segments are achieved by the cyclic controller by pushing forward and retracting the segments at velocities of $5.5 \mathrm{~mm}$ and $0.5 \mathrm{~mm}$ respectively, whilst still maintaining the overall net speed (defined at the mid point of all segment positions). A percentage pullback (how far each needle segment retracts during a cyclic motion) of approximately $30 \%$ is used, as this value was found to best minimize tissue strain and deformation in [11]. Other control parameters are summarized in Table I.

TABLE I

CONTROLLER PARAMETERS

\begin{tabular}{llcc}
\hline \hline Parameter & Symbol & Value & Unit \\
\hline Cyclic Period & $T_{c}$ & 2 & $\mathrm{~s}$ \\
Cyclic Frequency & $f$ & 0.5 & $\mathrm{~Hz}$ \\
Number of segments & $S e g_{\text {num }}$ & 4 & - \\
Minimum radius of curvature & $R$ & 70 & $\mathrm{~mm}$ \\
Net Velocity & Vel $_{\text {net }}$ & 1 & $\mathrm{~mm} / \mathrm{s}$ \\
Forward Velocity & Vel $_{\text {fwd }}$ & 5.5 & $\mathrm{~mm} / \mathrm{s}$ \\
Retraction Velocity & Vel $_{\text {ret }}$ & 0.5 & $\mathrm{~mm} / \mathrm{s}$ \\
Percentage Pullback & Pullback & 27 & $\%$ \\
Maximum Stroke Factor & $S_{f}$ & 50 & $\%$ \\
Maximum Stroke Length & $S_{\max }$ & 4.1 & $\mathrm{~mm}$ \\
Minimum Stroke Length & $S_{\min }$ & 1.4 & $\mathrm{~mm}$ \\
Segment Period Forward & $S P$ & 0.5 & $\mathrm{~s}$ \\
Segment Period Forward Max & $S P_{\max }$ & 0.75 & $\mathrm{~s}$ \\
Segment Period Forward Min & $S P_{\min }$ & 0.25 & $\mathrm{~s}$ \\
\hline \hline
\end{tabular}

After defining the cyclic period, segment velocities and maximum stroke factor, it is possible to define the maximum achievable offset by any one segment over the full cyclic controller period as in Eq. 1, resulting in a maximum achievable offset of $1.5 \mathrm{~mm}$ per cycle.

$$
\begin{aligned}
\text { Offset }_{\max }= & T_{c} \cdot f / 2 \cdot\left(\mathrm{Vel}_{f w d} \cdot S P_{\text {max }}+\mathrm{Vel}_{\text {ret }} \cdot S P_{\text {min }}\right) \\
& -T_{c} \cdot f / 2 \cdot\left(\mathrm{Vel}_{f w d} \cdot S P_{\text {min }}+\mathrm{Vel}_{\text {ret }} \cdot S P_{\text {max }}\right)
\end{aligned}
$$


Whilst the desired offset from the human operator is larger than this value, the maximum offset will be commanded, until the system can achieve the desired configuration within one cyclic period. New offset commands are only processed at the cyclic period of 2 seconds. The velocity profiles of each segment are determined in this way, and then offset in time from each other by $T c / S g_{\text {num }}=0.5 \mathrm{~s}$. This ensures the segments do not all move forward at the same time, but rather move cyclically with respect to one another. To generate the direct motion profile, the desired offset is achieved using the Forward Velocity. Once achieved, the velocity profile returns to the Net Velocity until a new offset command is processed at the same rate as that for the cyclic controller.

\section{RESULTS}

To test the performance of the controllers, a desired path with a double bend in 3D was generated at the limits of the maximum achievable needle curvature (before reaching buckling) to simulate the greatest change in needle configuration we can expect to see under real experimental conditions. The curvatures of the PTF $k_{1}, k_{2}\left(\mathrm{~mm}^{-1}\right)$ defined in the two otho-normal planes (respectively the $\mathrm{Y}$ and $\mathrm{Z}$ planes as in Fig. 3) were selected as:

$$
\begin{aligned}
& {\left[\begin{array}{ll}
k_{1} & k_{2}
\end{array}\right]=\left[\begin{array}{ll}
C_{Y} & C_{Z}
\end{array}\right]=[-0.0072,-0.0048 ;} \\
& 0.0143,0.0095]
\end{aligned}
$$

corresponding to radius of curvature $(\mathrm{R})(\mathrm{mm})$ of:

$$
\begin{aligned}
& {\left[\begin{array}{ll}
R_{Y} & R_{Z}
\end{array}\right]=[-139,-208 ;} \\
& 70,105]
\end{aligned}
$$

The inverse model resulted in relative desired offsets (DO) (mm) between the four segments of:

$$
\begin{gathered}
{\left[D O_{11}, D O_{12}, D O_{13}, D O_{14}\right]=[0,-10.3,-17.2,-6.9} \\
0,20.6,34.3,13.7]
\end{gathered}
$$

where the subscript corresponds to the segment numbers the offset is between. These commands are relative to the current needle configuration.
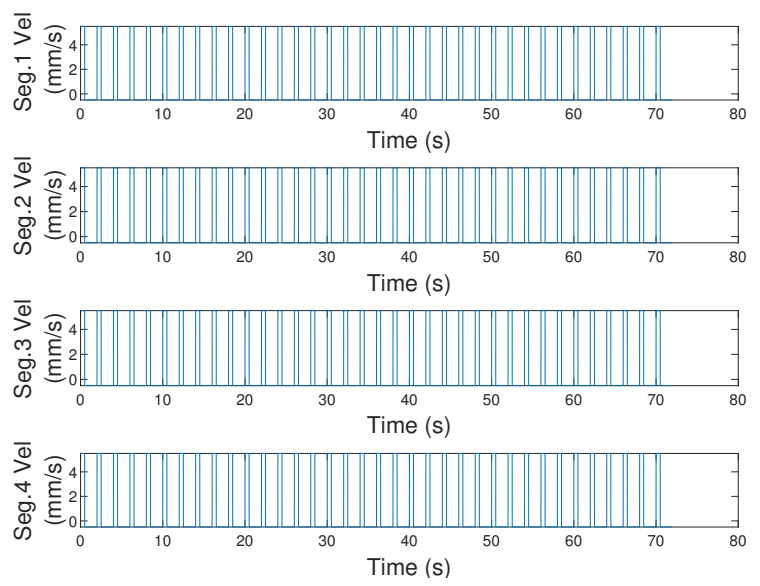

Fig. 4. Cyclic Controller velocity profile for each segment.
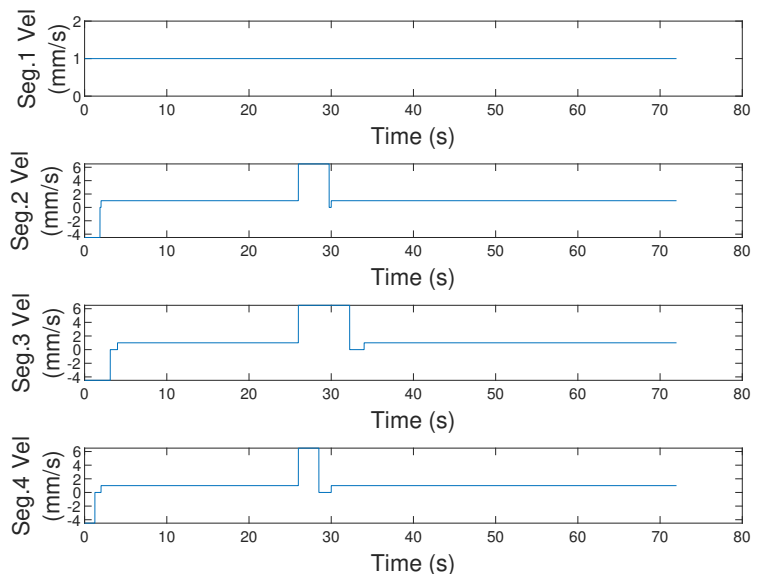

Fig. 5. Direct Controller velocity profile for each segment.

The corresponding velocity profiles for the cyclic and direct controllers are depicted in Figs. 4 and 5, respectively, and the displacement profiles for the cyclic and direct controllers in Figs 6 and 7, respectively.

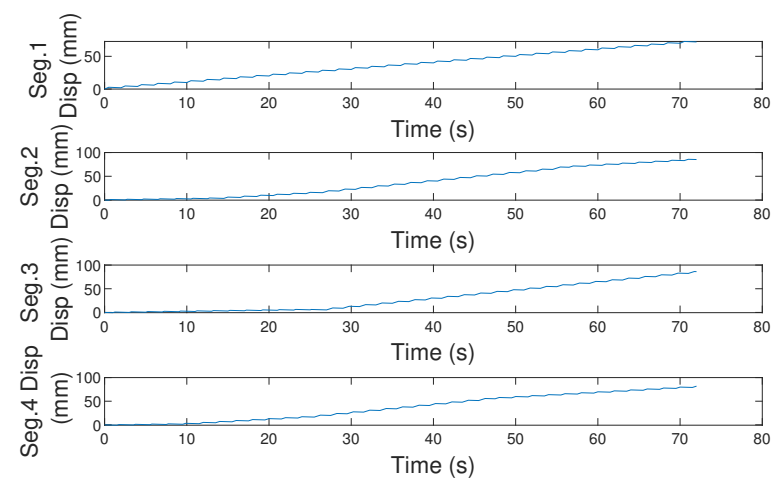

Fig. 6. Cyclic Controller displacement profile for each segment.

The trajectory taken by the needle over a total insertion length of $100 \mathrm{~mm}$ from the simulated forward model module for the two controllers is shown in Fig. 8. An insertion length of $100 \mathrm{~mm}$ was chosen as this is typical maximum length for a human neurosurgical procedure.

\section{DISCUSSION}

There are some important considerations to note from the velocity and displacement profiles. It takes a longer time for the cyclic controller to achieve the same offset configuration than that for the direct controller, due to the limitation of maximum achievable offset per cycle, as defined in Eq. 1, which does not apply to the direct controller. To demonstrate this effect, a single bend of maximum curvature was simulated and the displacement profiles of the controllers is shown in Fig. 10.

Indeed, for the commanded curvature and corresponding offset configuration of:

$$
\left[\begin{array}{ll}
C_{Y} & C_{Z}
\end{array}\right]=[0.0143,0.0143]
$$

$\left[D O_{11}, D O_{12}, D O_{13}, D O_{14}\right]=[0,20.59,41.19,20.59]$ 


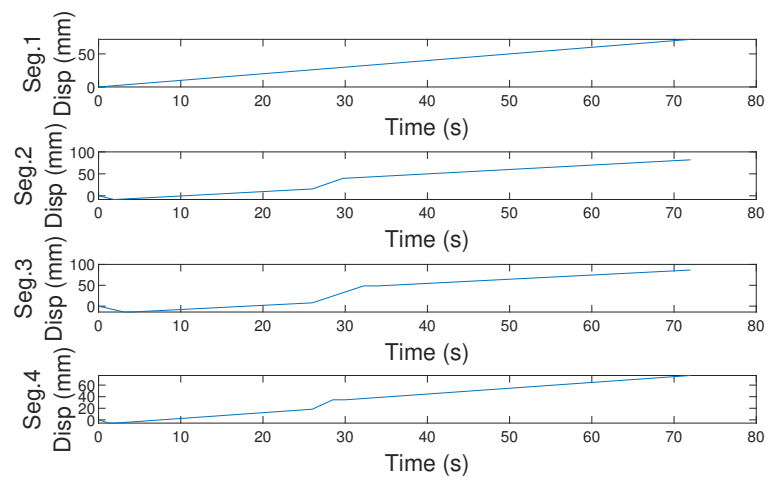

Fig. 7. Direct Controller displacement profile for each segment.

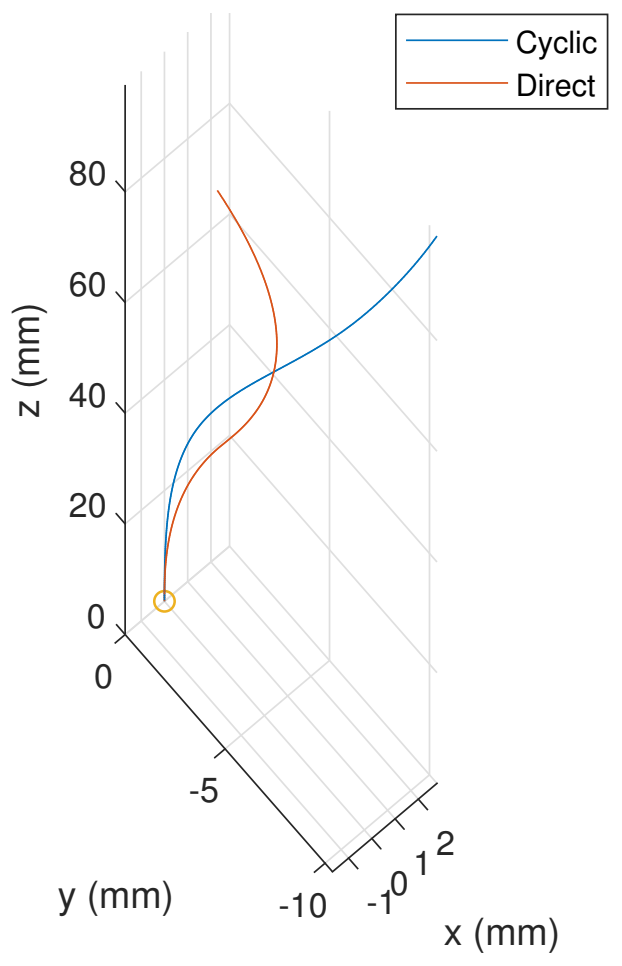

Fig. 8. Simulated trajectories of the low level controllers.

equivalent to the minimum achievable turning radius (or maximum curvature) of the needle of $70 \mathrm{~mm}$, it takes the direct controller 7.5 seconds to achieve the configuration, versus the 58 seconds it takes the cyclic controller. Note that the displacement offset at these time points is the required offset plus the displacement resulting from the net insertion speed of $1 \mathrm{~mm} / \mathrm{s}$. The cyclic controller is still able to achieve the necessary, maximum curvature, it just takes longer to do so. This means the path the needle follows to achieve the desired curvature is not as 'tight' as that for the direct controller, though once achieved it maintains the required turning radius as that for the direct controller. As the simulated net speed of the needle is constant, it is hypothesized that the cyclic controller will under-steer compared to the direct controller due to this response, and
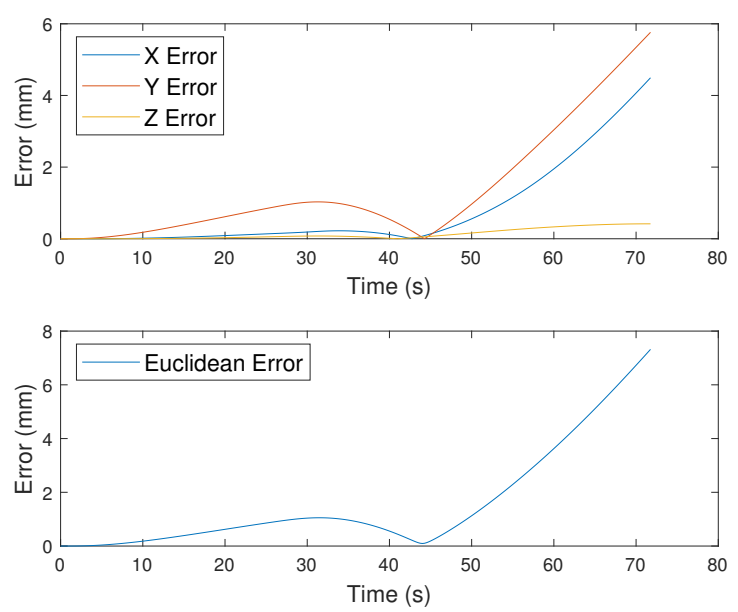

Fig. 9. Errors between the cyclic and direct controller trajectories.
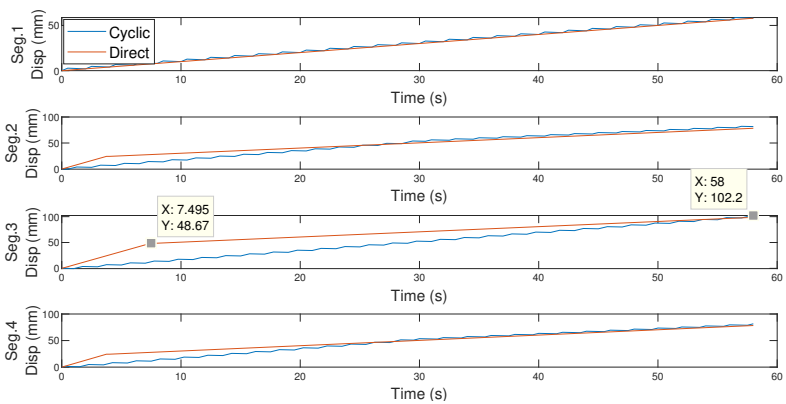

Fig. 10. Displacement profiles for a single bend from the cyclic and direct controllers, showing values reached when the desired offset was achieved for the direct controller (left) and cyclic controller (right) ignoring Segment 1 , which always has a desired offset of $0 \mathrm{~mm}$.

this is clearly seen in Fig. 8. The axes errors (top) and the Euclidean error (bottom) between the two paths is shown in Fig. 9. The error increases with the insertion length, as the cyclic controller is not able to achieve the desired curvature in the same time as the direct controller. This result is more visible in $3 \mathrm{D}$ steering, compared with $2 \mathrm{D}$ steering, as errors between the direct and cyclic trajectories are more pronounced when there is a change of plane of the steering arc.

\section{CONCLUSIONS \& FUTURE WORK}

The presented results highlight important areas of future controller design work. Given that a cyclic controller is desirable due to the lessened tissue strain it places on the brain, but has worse trajectory tracking performance as shown with this simulation study, future designs will need to incorporate a considered solution to the trade-off between insertion times and cyclic motion profiling. Possible design directions include to:

1) Develop a closed loop controller around the cyclic controller which can incorporate an adaptive speed profile depending on the desired configuration. 
2) Assume the human operator can correct the performance in real-time and keep the open loop cyclic controller design as is.

3) Use a combination of direct and cyclic motion profiles according to the magnitude of offset configuration change to benefit from the timely response of the direct controller and cyclic profiles from the cyclic controller.

Each of these design directions have different considerations to take into account - the first requires a non-constant insertion speed profile which may not be acceptable to neurosurgeons and increases the complexity of the low level controller, the second heavily relies on the operator's skill to adequately compensate the controller error (to be evaluated in future human study trials) and the third may result in actuation profiles where cyclic motion is not used, hence more strain is applied on the surrounding tissue. Future research and experiments, particularly under laboratory conditions using both phantom and ex-vivo tissue, will be required to quantify the performance and adequacy of the above design methodologies.

\section{ACKNOWLEDGMENTS}

The research leading to these results has received funding from the European Union's EU Research and Innovation Programme Horizon under grant agreement No 688279.

\section{REFERENCES}

[1] Richard L. Bishop. There is more than one way to frame a curve. The American Mathematical Monthly, 82(3):246-251, 1975.

[2] C. Burrows, R. Secoli, and Ferdinado Rodriguez y Baena. Experimental characterisation of a biologically inspired $3 \mathrm{~d}$ steering needle. In 2013 13th International Conference on Control, Automation and Systems (ICCAS 2013), pages 1252-1257, Oct 2013.

[3] Christopher Burrows, Fangde Liu, Alexander Leibinger, Riccardo Secoli, and Ferdinando Rodriguez y Baena. Multi-target Planar Needle Steering with a Bio-inspired Needle Design, pages 51-60. Springer International Publishing, Cham, 2017.

[4] Uroš Cerkvenik, Bram van de Straat, Sander W. S. Gussekloo, and Johan L. van Leeuwen. Mechanisms of ovipositor insertion and steering of a parasitic wasp. Proceedings of the National Academy of Sciences, 114(37):E7822-E7831, 2017.

[5] P. E. Dupont, J. Lock, B. Itkowitz, and E. Butler. Design and control of concentric-tube robots. IEEE Transactions on Robotics, 26(2):209225, April 2010.

[6] J. A. Engh, G. Podnar, D. Kondziolka, and C. N. Riviere. Toward effective needle steering in brain tissue. In 2006 International Conference of the IEEE Engineering in Medicine and Biology Society, pages 559-562, Aug 2006.

[7] L Frasson, S Y Ko, A Turner, T Parittotokkaporn, J F Vincent, and F Rodriguez y Baena. Sting: a soft-tissue intervention and neurosurgical guide to access deep brain lesions through curved trajectories. Proceedings of the Institution of Mechanical Engineers, Part H: Journal of Engineering in Medicine, 224(6):775-788, 2010. PMID: 20608494
[8] Felix Orlando Maria Joseph, Madhukar Kumar, Karly Franz, Bardia Konh, Parsaoran Hutapea, Yan-Jiang Zhao, Adam P Dicker, Yan Yu, and Tarun K Podder. Control of shape memory alloy actuated flexible needle using multimodal sensory feedbacks. Journal of Automation and Control Engineering Vol, 3(5), 2015.

[9] L. Kauhanen, T. Palomäki, P. Jylänki, F. Aloise, Marnix Nuttin, and José del R. Millán. Haptic feedback compared with visual feedback for bci. In Proceedings of the 3rd International Brain-Computer Interface Workshop and Training Course 2006, Graz, Austria, 92006.

[10] S. Y. Ko, L. Frasson, and F. Rodriguez y Baena. Closed-loop planar motion control of a steerable probe with a "programmable bevel"; inspired by nature. IEEE Transactions on Robotics, 27(5):970-983, Oct 2011.

[11] Alexander Leibinger, Matthew J. Oldfield, and Ferdinando Rodriguez y Baena. Minimally disruptive needle insertion: a biologically inspired solution. Interface Focus, 6(3), 2016.

[12] A. Majewicz, J. J. Siegel, A. A. Stanley, and A. M. Okamura. Design and evaluation of duty-cycling steering algorithms for roboticallydriven steerable needles. In 2014 IEEE International Conference on Robotics and Automation (ICRA), pages 5883-5888, May 2014.

[13] Panadda Marayong and Allison M. Okamura. Speed-accuracy characteristics of human-machine cooperative manipulation using virtual fixtures with variable admittance. Human Factors, 46(3):518-532, 2004.

[14] Eloise Matheson, Riccardo Secoli, Christopher Burrows, Alexander Leibinger, and Ferdinando Rodriguez y Baena. Cyclic motion control for programmable bevel-tip needles to reduce tissue deformation. Journal of Medical Robotics Research, 2018.

[15] Andrew Palisch, Raj G. Patel, Christina Gutowski, Adam C. Zoga, Phillip Colucci, Brian J. O'Hara, Catherine C. Roberts, and John Abraham. Analysis of needle type for musculoskeletal lesion biopsy: results of a novel steerable needle. Current Orthopaedic Practice, 27(4), 2016

[16] Marta Scali, Tim P Pusch, Paul Breedveld, and Dimitra Dodou. Needle-like instruments for steering through solid organs: A review of the scientific and patent literature. Proceedings of the Institution of Mechanical Engineers, Part H: Journal of Engineering in Medicine, 0(0):0954411916672149, 2017.

[17] R Secoli and F Rodriguez y Baena. Adaptive path-following control for bio-inspired steerable needles. In 2016 6th IEEE International Conference on Biomedical Robotics and Biomechatronics (BioRob), pages 87-93, June 2016.

[18] N. J. van de Berg, D. J. van Gerwen, J. Dankelman, and J. J. van den Dobbelsteen. Design choices in needle steering; a review. IEEE/ASME Transactions on Mechatronics, 20(5):2172-2183, Oct 2015.

[19] Thomas Watts, Riccardo Secoli, and Ferdinando Rodriguez y Baena. Modelling the deformation of biologically inspired flexible structures for needle steering. In Mechanism, Machine, Robotics and Mechatronics Sciences: Proceedings of the First International Congress for the Advancement of Mechanism, Machine, Robotics and Mechatronics Sciences (ICAMMRMS-2017), 2017.

[20] R. J. Webster, A. M. Okamura, and N. J. Cowan. Toward active cannulas: Miniature snake-like surgical robots. In 2006 IEEE/RSJ International Conference on Intelligent Robots and Systems, pages 2857-2863, Oct 2006

[21] E. Westebring, P. van der Putten, R. H. M. Goossens, J. J. Jakimowicz, and J. Dankelman. Haptics in minimally invasive surgery - a review. Minimally Invasive Therapy and Allied Technologies, 17(1):3-16, 2008 